\title{
Level Shifting based Decision Trimmed Filter for Multiplicative Noises
}

\author{
Sukhninder Singh \\ Department of ECE, \\ Beant College of Engineering and Technology, \\ Gurdaspur (Pb.), India.
}

\author{
Neetu \\ Department of ECE, Beant \\ College of Engineering and Technology, \\ Gurdaspur (Pb.), India.
}

\begin{abstract}
The most recent approach manages to boost the particular contrast within digital images in productive manner through the use of the incorporated proposed algorithm. To preserve the edges and borders gradient based smoothing will also be used. And also the proposed approach has offered quite useful results, thus causes it to become more useful. The planned technique is created and implemented inside the MATLAB utilizing image processing toolbox. The comparison among proposed and existing median filter techniques will also be drawn by taking some image performance parameters like root mean square error, edge preservation index.
\end{abstract}

\section{Keywords}

Digital Image Processing, Applications of image processing, filters

\section{INTRODUCTION}

Digital Image Processing (DIP) is an integral part of digital signal processing. The area of DIP identifies dealing with digital images through an electronic digital computer [1]. Digital Picture Processing requires the difference of digital knowledge for increasing the qualities of picture with the help of computer. An electronic picture is a display of a twodimensional picture as a finite set of digital prices, known as picture things or pixels. The digital picture contains a set number of lines and articles of pixels. The digital picture is provided as insight in to a computer and computer is set to improve these knowledge with the aid of an equation or with number of equations and then store the prices of the computation for each pixel or picture aspect [1]. Digital picture processing has several benefits in terms of price, pace and edibility etc. Digital picture processing assists one to boost picture top features of curiosity while attenuating depth unrelated to certain program, and then extract useful data regarding the world from the increased image.

\subsection{Light}

Other samples of electromagnetic radiation are gamma radiation, UV and infra-red radiation and radio dunes, and all electromagnetic radiation journey at the pace of gentle $(3 \times$ $108 \mathrm{~m} / \mathrm{s}$ ).

Electromagnetic radiation contains dunes and may be reflected and refracted the same as noise dunes (but noise dunes aren't electromagnetic waves). We can only see items that emit gentle, and you will find two ways that this will happen. The item may emit gentle itself, like a light or a computer check, or it shows gentle that falls on it. An object that shows gentle usually absorbs gentle as well. When we see the item as red it indicates that the item absorbs all gentle except red, which is reflected. An object that produces gentle is significantly different; if it's to be perceived to be red it must emit only red light.

\subsection{Digital output media}

Our focus will soon be on items that emit gentle, for example a computer display. A pc check includes a square variety of small dots which emit light. In many systems, each dot is actually three smaller dots, and all these smaller dots emit RGB color light. If the amount of red, green and blue is varied, our mind merges the gentle from the three small gentle options and perceives gentle of various colors. In this way the color at each set of three dots could be controlled, and a shade image could be developed from the full total amount of dots.

It is important to realize that it is probable to create many, but not all, shades by mixing red, green and blue. Additionally, various computer displays use slightly various red, green and blue shades, and unless this really is taken into consideration, shades will look various on the 2 monitors. This also ensures that some shades that can be exhibited using one check might not be displayable on an alternative monitor. Models utilize the same concept of building a picture from small dots. Of all printers but, the small dots do not contain smaller dots of various colors. Alternatively as much as $7-8$ various inks (or related substances) are combined to the proper color. That afford them the ability to generate a wide range of shades, but not all, and the situation of corresponding a shade from still another product just like a check is at least as hard as corresponding various shades across various monitors. Movie projectors develop a picture that is estimated onto a wall. The final image is thus a reflected image and it is important that the surface is white such that it shows all shades equally. The grade of a computer device is strongly connected to the thickness of the dots.

\subsection{Digital input media}

The two most typical ways to obtain electronic photos has been a digicam or even a scanner. A protection essentially requires a photograph of a report in the shape of a square variety of (possibly colored) dots. For units, a significant way of measuring quality is the number of dots per inch. For cameras it doesn't make sense to measure the resolution in dots per inch, as this depends on how the image is produced (its size). As an alternative the resolution is assessed in the number of dots recorded.

For scanners and cameras it is simple to think that the more dots (pixels), the greater the quality. Though there's some reality to this, there are many different facets that effect the quality. The main problem is that the assessed shade information is quickly polluted by noise. And obviously high res entails that the resulting documents become very huge; an uncompressed $6000 \times 4000$ image generates a $72 \mathrm{MB}$ file. The main advantage of high res is that you could magnify the image significantly and however maintain realistic quality. 


\subsection{Definition of digital image}

From the mathematical viewpoint, a visual is very simple. The price allows along with data at the reason $(i, j)$. It is important to think about that there are many models for this. The simplest condition is simple dark and brilliant photos where condition is both 0 and 1 . For grey-level photos the intensities tend to be integers in the merchandise selection 0-255. However, we shall believe the intensities differ in the period $[0,1]$, as this often simplifies the shape of some mathematical functions.

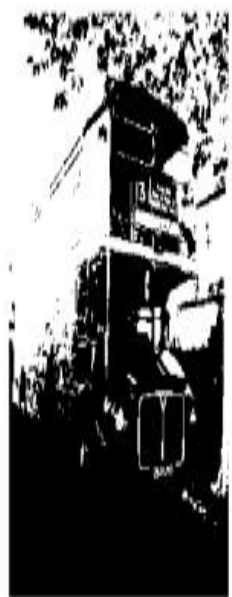

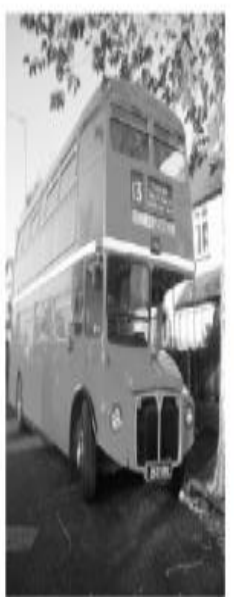

(b)

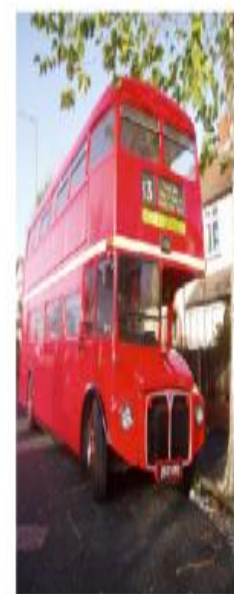

Fig.1. various of the same picture; dark and bright (a), grey-level (b), and colour (c).

\section{APPLICATIONS OF IMAGE PROCESSING}

\section{a) X-Ray Imaging}

$\mathrm{X}$-Ray is one of the earliest supply of EM radiation used for imaging. X-Ray is used in medical diagnostics. Yet another use of X-Ray in medical imaging is electronic axial to zoography.

\section{b) Imaging in extremely purple Group}

Ultra purple is used in fluorescence microscopy.

\section{c) Digicam photographs}

Cameras have digital image control pieces which are used to adjust row information from picture indicator into shade corrected picture in a regular picture file structure. The photos collected from cameras are extra processes to development the quality.

\section{d) Intelligent Transport Process}

Digita1 image control has extensive role in transport program like quantity menu recognition.

\section{e) Electron Microscopy}

It runs on the microscope that may boost very moment detai1s with very excessive handling power.

\section{f) Ultra sound}

It's used for medical function wherever excessive frequency breed bead sound dunes are returned by tissue

\section{FILTER}

Filter in an image managing can be a foundation purpose that is placed on achieves several jobs such as for example as an example sound decrease [5], interpolation, and re-sampling. Filter photograph data can be a normal method employed in nearly all photograph managing systems. Your decision of filter is determined by the type of the duty done by filter and perform and kind of the data. Filters are comfortable to remove sound from electronic photograph while maintaining the facts of photograph preserved is a necessary section of photograph processing.

Many images are affected to some extent by noise that is mysterious difference in data: disturbances in image power which are possibly uninterruptable or perhaps not of interest. Picture evaluation is usually simplified if this noise could be blocked out. Within an similar way filters are used in chemistry to free drinks from stopped impurities by driving them by way of a layer of sand or charcoal. Technicians employed in indicate control have lengthy this is of the term filter to include procedures which intensify features of interest in data. Hiring this broader explanation, image filters may be used to highlight sides - that is, boundaries between things or elements of things in images. Filters provide assistance to visible interpretation of images, and can also be applied as a precursor to help electronic control, such as segmentation. Filters change a pixel's price considering the values of neighboring pixels too. They may possibly be used straight to noted images. A show of the output from the $5 \times 5$ going normal filter. Each pixel has been replaced by the average of pixel values in a $5 \times 5 \mathrm{sq}$, or window centered on that pixel. The result is to cut back noise in the image, but also to cloud the sides of the fibers.

\subsection{Median filter}

Median filter is the type of nonlinear filter through which we obtain the median cost to displace the noisy pixel. Averaging is certain to generate a greater estimate of $I[x ; y]$ when the typical is bought out a homogeneous town with zero-mean noise. When the location straddles the boundary between two such pieces, the estimate employs products from two energy populations, resulting in blurring of the boundary.

A popular change may be the median filter, which changes a pixel price with the median price of the neighborhood. the median filter can clean loud pieces however greater protect the construction of boundaries between them. When a pixel is really selected from on the list of bright sections, but shut sideways, it's potential that many the values inside their neighborhood are bright pixels with sound: if this is correct, then neighboring pixels from a black square won't be properly used to discover the productivity value.

\subsection{Sorted Switching Median Filter}

Sorted Switching Median Filter (i.e.SSMF) for effectively denoising exceptionally broken images while maintaining the image details. In the sensing time it'll conduct histogrambased intuition noise recognition to recognize the locations of potential broken pixels. The choosing time can rely the amount of uncorrupted pixels for this broken pixel. The selection time works on the reduce median filter to revive a lot of the corrupted. Gaussian filters are the only real forms which are separable and, at the very least to a lattice approximation, circularly symmetric. Moreover they over come another mentioned problem of going usual filter since loads corrosion to zero.

\subsection{Mean Filter}

The mean filter is just a simple spatial filter. It is just a sliding-window filter that changes the center price in the window. It changes with the common mean of all the pixel values in the kernel or window. The screen is generally square but it can be of any shape. 


\subsection{Wiener Filter}

The objective of the Wiener filtration would be to filter the noise that's broken a signal. This filtration is dependent on a statistical approach. Primarily all the filters were created for a desired volume response. Wiener filtration copes with the filter of an mage from a different view. This filtration is capable of lowering the noise and degrading function. One method that we assume we've understanding of the spectral house of the noise and unique signal. We applied the Linear Time Invariant filtration gives output similar regarding the unique signal just as much probable.

\section{RELATED WORK}

Chengbin Zhang et al. 2015 [1] described a whole new algorithm to get rid of intuition sound from highly damaged images. That strategy cannot just achieve greater picture quality, but likewise have smaller computation time. And this process is simple and simple to be understood. Simulation effects reveal that this process provides greater performance than the existing strategy presented for damaged by highly sound thickness when it comes to sound decrease and depth preservation. That strategy provides an effective filter human anatomy -accomplish and is fantastic for real-time implementation because it operates on the set $3 \times 3$ window rating for filter processing.

S. Esakkirajan et al. 2011 [2] described a fresh algorithm Modified Decision Centered Unsymmetric Connected Median Purification (MDBUTMF) provides greater performance in comparison with provide sound elimination calculations when it comes to PSNR and IEF. The performance of the algorithm has been tried at different sound densities on similarly grayscale and shade images. Also at big sound thickness levels the MDBUTMF provides greater advantages in comparison with different provide algorithms. Equally visible and quantitative responses are demonstrated. The algorithm is beneficial for sodium and pepper sound elimination in images at big sound densities. The algorithm practices the damaged images by first detecting the intuition noise. The operating pixel is examined whether it's loud or loud free. That is, if the handling pixel lies between optimum and little gray level rates then its sound free pixel, it's remaining unchanged. If the operating pixel wants the absolute most or little gray level then it's loud pixel which can be usually structured by MDBUTMF.

Priyanka Kamboj et al. 2013 [3] described that progress of a loud picture is very important job in electronic picture processing. Filters are applied most readily useful for reducing sound from the images. Various types of sound versions and filters practices have now been discussed. Filters practices are split into two aspects linear and non-linear methods. Subsequent learning linear and non-linear filter each of have restricts and advantages. In the corner filter systems, you can find several filters are advised to filter a broken location. The main decision is to use a particular filter is on the cornerstone of the various sound level at the many test pixel place or performance of the filter scheme on a filter mask.

Shanmugavadivu et al. 2012 [4] planned a filter which can be much better in solving the images damaged with fixedvalue intuition noise. Because the planned filter is computationally simple, the restoration charge is faster. That filter finds program in reducing sound from numerous reading images, present in the research of area morphology, since these images are invariably transformed by set cost intuition noise.
Shanmugavadivu et al. 2011 [5] stated a lately developed sound filter particularly, Adaptive Two-Stage Median Purification (ATSM) to denoise the images damaged by fixedvalue intuition noise. The performance of the planned filter is turned out to be greater when it comes to Prime Signal-toNoise Proportion and human visible perception. That filter is effectual in denoising the highly damaged image.

K. S. Srinivasan et al. 2007 [6] shown a whole new decisionbased algorithm for restoration of pictures which can be very broken by wish noise. The most recent algorithm reveals considerably higher display quality in comparison with a Common Median Filter and different nonlinear filters. The planned method, unlike different nonlinear filters, removes only broken pixel by the median value or by its neighboring pixel value. The algorithm is tested on different different pictures and is build to produce higher results in terms of the qualitative and quantitative methods of the image.

V. Jayaraj et al. 2010 [7] determined the newest process which presents the notion of alternative of noisy pixels by linear forecast ahead of estimation. A story simplified linear predictor is created with this particular purpose. The goal of the system and algorithm is the removal of high-density salt and pepper noise in images. The newest algorithm shows significantly greater picture quality with great PSNR, compensated down MSE, and great edge preservation.

K. Aiswarya et al. 2010 [8] determined a brand new algorithm to get rid of high-density salt and pepper noise using modified sheer selecting method. The newest algorithm has reduced computation time when compared to different normal algorithms. Outcomes of the algorithm are in distinction to different provide calculations and it's shown that the newest process has greater aesthetic look and quantitative actions at higher noise densities. The algorithm is founded on a brand new idea of alternative ahead of opinion in contrast to the typical switching-based nonlinear filters.

S.J Horng et al. 2013 [9] determined the newest process which uses a detecting time, selecting time and filtration time to effectively recover very corrupted images. That filtration is as simple-of-the-art changing filters. It is really suited to many real-time photograph running applications. Generally, that filtration is able to restrain hope noise about $95 \%$ noise occurrence level. Significant simulation effects disclose that filtration constantly outperformed different filters under different noise versions by attaining greater PSNR across a wide selection of noise densities. That filtration offers a large profit of progress at very high noise occurrence corruption.

P. E. Ng et al. 2006 [10] planned a book changing median filtration introducing with a strong hope noise recognition process for effectively denoising acutely corrupted images. To ascertain whether the prevailing pixel is corrupted, the algorithm first classifies the pixels of a local screen, centering on the prevailing pixel, into three groups-lower level hope noise, uncorrupted pixels, and higher level hope noise. Significant simulation effects conducted on pictures under a wide selection of noise issue obviously demonstrate that changing median filtration considerably outperforms all provide median-based filters, with regards to controlling hope noise while keeping photograph facts, and is algorithmically simple, suitable for real-time implementation and application.

Zinat Afrose et al. 2012 [11] defined a method to remove Salt \& pepper, Gaussian and Speckle noise from element pictures using median filtration, calm median filtration, wiener, center measured median and averaging filtration. The 
performance of the different filters with the used tones using element pictures are compared and reviewed based on PSNR value. From the performance analyses the calm median filtration enables greater advantages for element images.

Wei Ping et al. 2007 [12] planned an easy and trusted behavioral wish noise filtration created for excessively broken photos. The planned filtration use connected system like BDND; it's furthermore very efficient and also better than BDND. The planned approach utilize a brand new successful along with successful noise recognition process to find out if new pixel have today been broken by tones, if it's harmful, a variable eye-port median filtration is used to attenuate behavioral wish noise, while the individuals uncorrupted pixels are keep unchanged.

\section{METHODOLOGY}

\subsection{Proposed Algorithm}

Following are the various steps required to successfully simulate the proposed algorithm

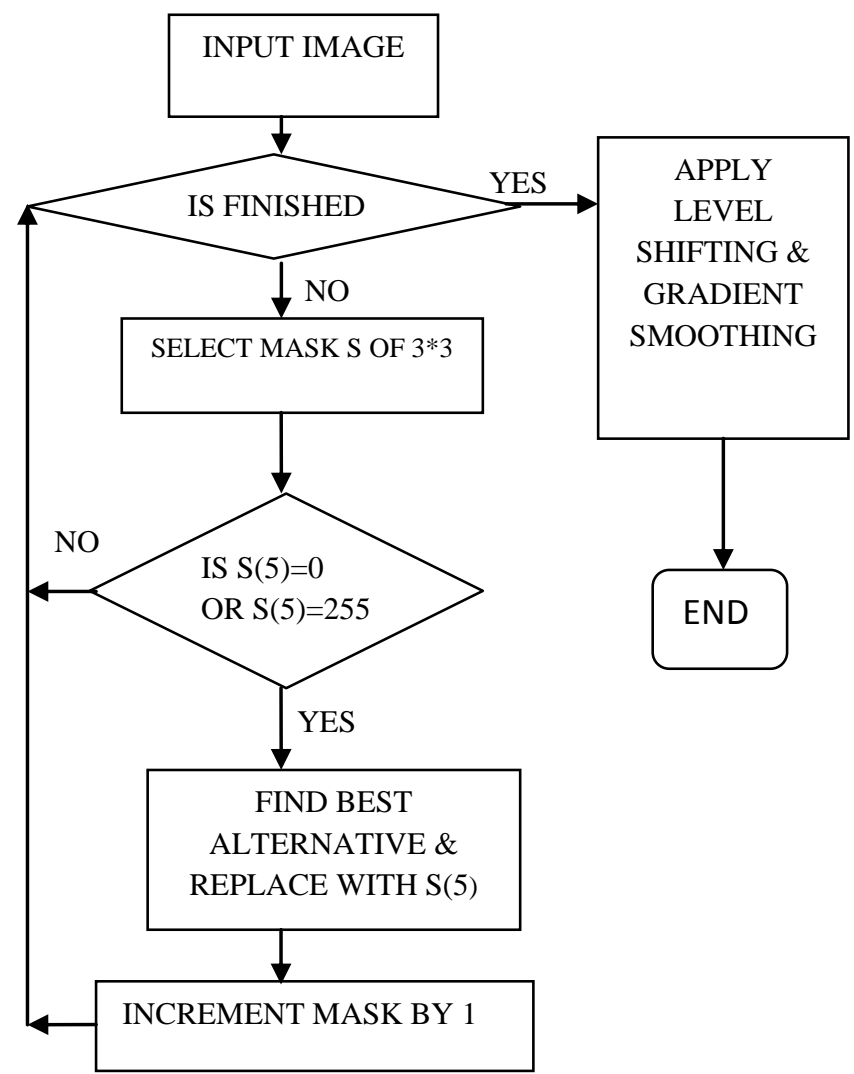

Fig 2: Flow Chart of proposed algorithm

The Proposed method is divided into two parts:

1) Algorithm will evaluate the centre pixel's value i.e. whether or not it is equal to 0 or 255 if yes then it will go to find the alternative noise free value for the same based on the multiple selection policy; else window will switched further.

2) This part will find the neighbourhood pixels of the center value and see whether all are having 0 or 255 as its value or not. If no then median will be evaluated and replaced with center value and window will switched further else it will use decision tree to evaluate the noise free value by taking the global median and global mean and also recently evaluated median to replace the same.

\section{RESULT AND DISCUSSION}
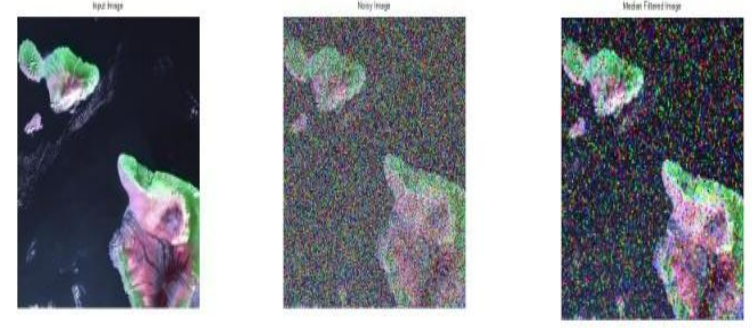

a) Input Image4

b) Noisy Image

c) Median Filter
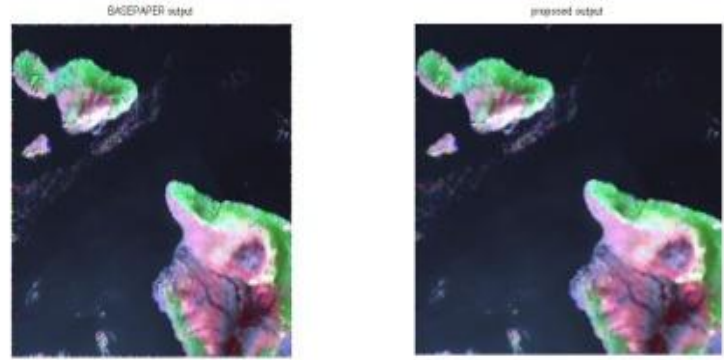

d) Base paper Results

e) Proposed Results

Fig 3: Experimental Results of image 4

The experimental results are taken under MATLAB version 2013. Firstly results are taken at noise density 0.6 in fig 3 .

a) Input Image - Any color input image is taken for experimental purposes.

b) Noisy Image - Now the 0.6 noise density is added in the input image.

c) Median Filter Image - This result shows the output of median filter.

d) Base-paper Results - This result shows the output of existing algorithm.

e) Proposed Results - This result shows the output of proposed algorithm.

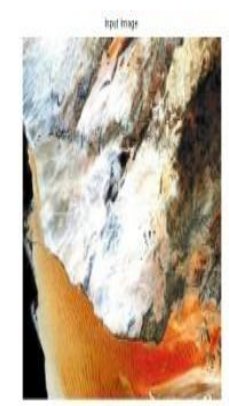

a)Input image5

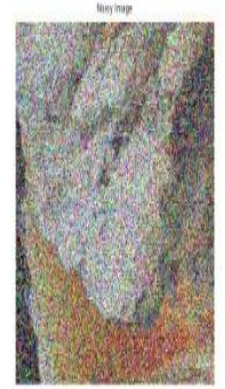

b) Noisy Image

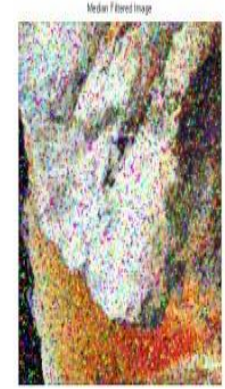

c) Median Filter 


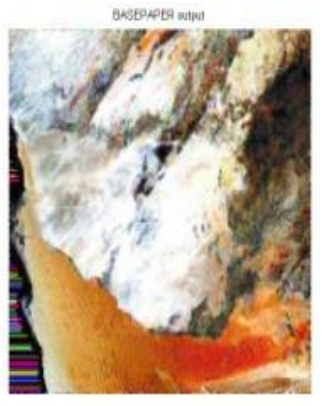

d) Basepaper Results

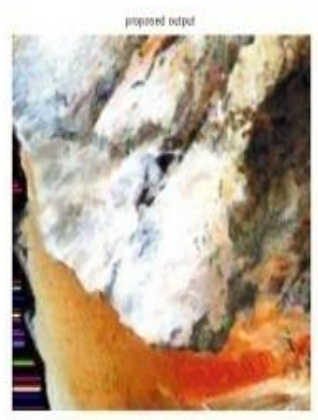

e) Proposed Results
Fig 4: Experimental Results of image 5

\section{PERFORMANCE ANALYSIS}

\subsection{EPI}

When we need to preserve edge information and at the same time preserve the edges. Even when uniform smoothing does not remove the boundaries, it does distort them. This is not acceptable in the context of, for example, medical imaging.

Table 1: Epi Comparison Table

\begin{tabular}{|l|l|l|l|l|}
\hline $\begin{array}{l}\text { Input } \\
\text { Images }\end{array}$ & $\begin{array}{l}\text { Noisy } \\
\text { Images }\end{array}$ & $\begin{array}{l}\text { Median } \\
\text { Filter }\end{array}$ & $\begin{array}{l}\text { Basepaper } \\
\text { Results }\end{array}$ & $\begin{array}{l}\text { Proposed } \\
\text { Results }\end{array}$ \\
\hline $\begin{array}{l}\text { Input } \\
\text { image1 }\end{array}$ & 0.2966 & 0.4549 & 0.9364 & 0.9569 \\
\hline $\begin{array}{l}\text { Input } \\
\text { image2 }\end{array}$ & 0.2582 & 0.4226 & 0.8967 & 0.8988 \\
\hline $\begin{array}{l}\text { Input } \\
\text { image3 }\end{array}$ & 0.5811 & 0.2645 & 0.9157 & 0.9298 \\
\hline $\begin{array}{l}\text { Input } \\
\text { image4 }\end{array}$ & 0.4840 & 0.3166 & 0.8575 & 0.8957 \\
\hline $\begin{array}{l}\text { Input } \\
\text { image5 }\end{array}$ & 0.4890 & 0.3084 & 0.8463 & 0.8763 \\
\hline $\begin{array}{l}\text { Input } \\
\text { image6 }\end{array}$ & 0.3895 & 0.3784 & 0.9162 & 0.9242 \\
\hline
\end{tabular}

This table 1 shows the EPI comparison results of noisy image, median filter image, basepaper results and proposed results.

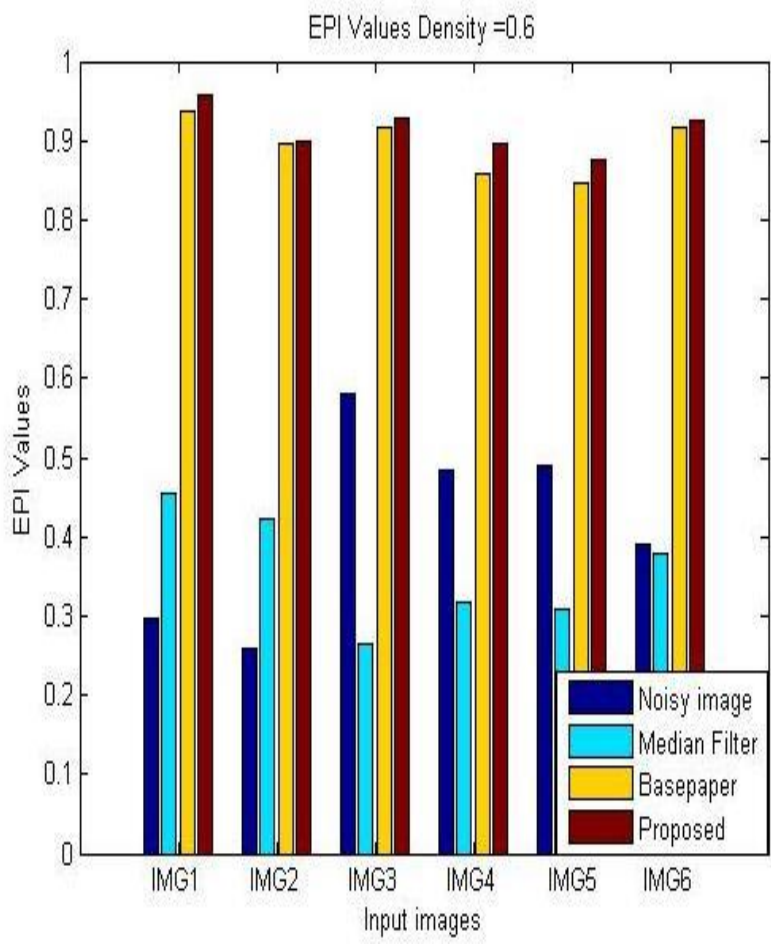

Fig 5: EPI Graph

This graphs depicts that proposed are much better (i.e. low) than the existing results which is as shown in fig 5 .

\subsection{RMSE (Root Mean Square Error)}

Root-mean-square error is a measure of the differences between values predicted by a model or an estimator and the values actually observed. It can be explained as:

RMSE $=\sqrt{\frac{1}{M N} \sum_{i=1}^{M} \sum_{j=1}^{N}\left(f(i, j)-f^{\prime}(i, j)\right)^{2}}$

Table 2: RMSE Comparison Table

\begin{tabular}{|l|l|l|l|l|}
\hline $\begin{array}{l}\text { Input } \\
\text { Images }\end{array}$ & $\begin{array}{l}\text { Noisy } \\
\text { Images }\end{array}$ & $\begin{array}{l}\text { Median } \\
\text { Filter }\end{array}$ & $\begin{array}{l}\text { Basepaper } \\
\text { Results }\end{array}$ & $\begin{array}{l}\text { Proposed } \\
\text { Results }\end{array}$ \\
\hline $\begin{array}{l}\text { Input } \\
\text { image1 }\end{array}$ & 107.3499 & 63.055 & 20.2237 & 16.5529 \\
\hline $\begin{array}{l}\text { Input } \\
\text { image2 }\end{array}$ & 105.0476 & 65.1767 & 26.0192 & 25.0576 \\
\hline $\begin{array}{l}\text { Input } \\
\text { image3 }\end{array}$ & 125.3316 & 74.2294 & 23.4734 & 21.4942 \\
\hline $\begin{array}{l}\text { Input } \\
\text { image4 }\end{array}$ & 120.8056 & 70.4557 & 19.1311 & 15.5242 \\
\hline $\begin{array}{l}\text { Input } \\
\text { image5 }\end{array}$ & 119.1176 & 71.8262 & 31.0966 & 27.3313 \\
\hline $\begin{array}{l}\text { Input } \\
\text { image6 }\end{array}$ & 112.9912 & 68.3811 & 24.1868 & 22.8473 \\
\hline
\end{tabular}

This table 2 shows the RMSE comparison results of noisy image, median filter image, basepaper results and proposed results. 


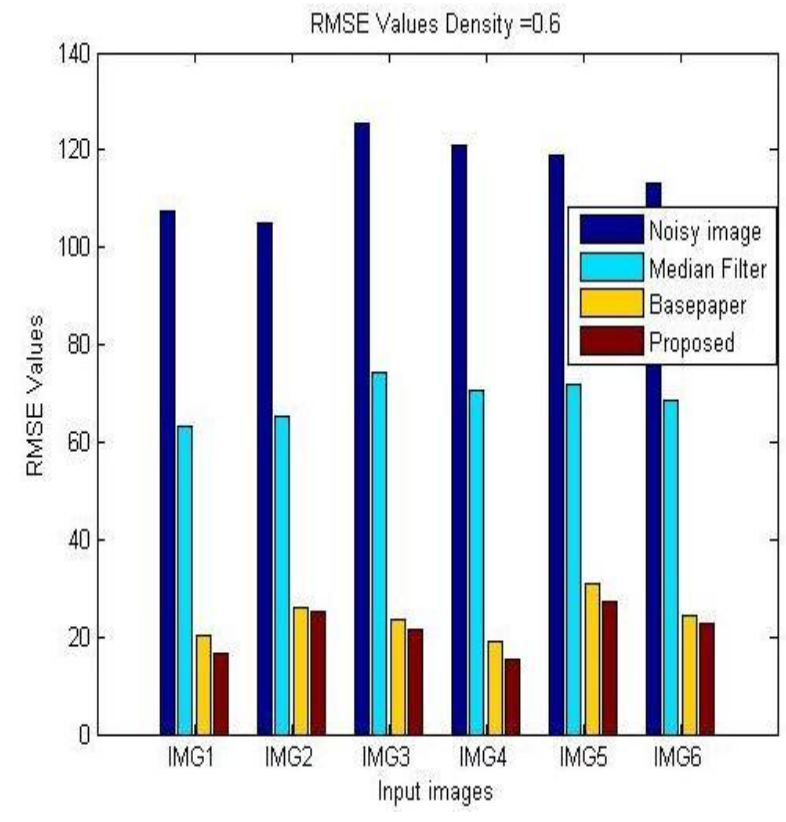

Fig 6: RMSE Graph

This graphs depicts that proposed are much better (i.e. low) than the existing results which is as shown in fig 6 .

\section{CONCLUSION}

The most recent approach are able to boost the particular contrast within digital images in useful manner by making use of the incorporated proposed enhancement algorithm. As edge preservation primarily based gradient based smoothing has capacity to reduce the actual effectuation connected with noise as well as it maintains the edges in successful manner therefore provides superior results. And also the proposed approach has offered quite effective results, thus can make it more realistic.

The proposed technique is created and implemented within the MATLAB employing image processing toolbox. The several kind regarding images has additionally been considered for trial purpose. Also distinct quality metrics also thought to evaluate the potency of the proposed technique. The comparison between existing along with the proposed approach has definitely shown that this proposed method outperforms within the available approaches.

\section{REFERENCES}

[1] Chengbin Zhang,Kaifu Wang, "A switching medianmean filter for removal of high density impulse noise from digital images," in Optik-Int. Jour. for light and electron optics (IJLEO-Vol. 126), 2015.
[2] S. Esakkirajan, T. Veerakumar, Adabala N. Subramanyam, and C. H. Prem Chand,"Removal of High Density Salt and Pepper Noise Through Modified Decision Based UnsymmetricTrimmed Median Filter", IEEE SIGNAL PROCESSING LETTERS, VOL. 18, NO. 5, MAY 2011

[3] Priyanka Kamboj, Versha Rani,'Image Enhancement Using Hybrid FilteringTechniques “,International Journal of Science and Research, Vol 2, No. 6, June 2013.

[4] Shanmugavadivu, Eliahim Jeevaraj,"Laplace Equation based Adaptive Median Filter for Highly Corrupted Images"International Conference on Computer Communication and Informatics 2012, Jan. $10-12$, 2012.

[5] Shanmugavadivu P and Eliahim Jeevaraj P S," FixedValue Impulse Noise Suppression for Images using PDE based Adaptive Two-Stage Median Filter", ICCCET-11 (IEEE Explore), pp. 290-295, 2011.

[6] K. S. Srinivasan and D. Ebenezer, "A new fast and efficient decision based algorithm for removal of high density impulse noise," IEEE Signal Process. Lett, vol. 14, no. 3, pp. 189-192, Mar. 2007.

[7] V Jayaraj and D. Ebenezer, "A new switching-based median filtering scheme and algorithm for removal of high-density salt and pepper noise in image," EURASIP J. Adv. Signal Process. 2010.

[8] K. Aiswarya, V. Jayaraj, and D. Ebenezer, "A new and efficient algorithm for the removal of high density salt and pepper noise in images and videos," in Second Int. Conf. Computer Modeling and Simulation,2010, pp. 409-413.

[9] S.J Horng,L.Y Hsu, "Using sorted switching median filter to remove high-density impulse noise," JVCIR,Vol 24,Oct 2013.

[10] P. E. Ng and K. K. Ma, "A switching median filter with boundary discriminative noise detection for extremely corrupted images," IEEE Trans. Image Process. , vol. 15, no. 6, pp. 1506-1516, June 2006.

[11] Afrose,"Relaxed Median Filter: A Better Noise Removal Filter for Compound Images", International Journal on Computer Science and Engineering (IJCSE) Vol. 4 No. 07 July 2011.

[12] Wei Ping et al., "A Fast and reliable switching median filter for highly corrupted images by impulse noise." vol. 1, pp. 4244-0921, IEEE, 2007. 